\title{
Influence of bone anisotropy on reliability assessment of mini-plate fixation system stabilization in symphysis mandibular fractures: Two studied cases under convalescence period
}

\author{
Influence de l'anisotropie osseuse sur l'évaluation de la fiabilité de la \\ stabilisation du système de fixation par mini-plaques dans les fractures \\ mandibulaires de la symphyse : Deux cas étudiés sous la période de la \\ guérison
}

\author{
Kharmanda G. ${ }^{{ }^{*}}$; Kharma M-Y. ${ }^{2}$; El-Hami A. ${ }^{1}$ \\ ${ }^{1}$ Mechanics Laboratory of Normandy, INSA Rouen, France \\ ${ }^{2}$ Al-Farabi College for Dentistry, Jeddah, Saudi Arabia \\ *Corresponding author: mgk@scs-net.org
}

\begin{abstract}
The reliability analysis is used to in order to measure the stability of the mini-plate fixation system used in the human fractured mandibles after the chirurgical operation. The failure is assumed to take place when the Most Probable failure Point (MPP) is found. In this work, two studied cases of 3-dimensional finite element models are considered for the same fracture situation. A successful fracture healing requires that a number of constraints which are influenced by the loading conditions are fulfilled, and since muscle activity is difficult to evaluate, there is a strong need to introduce loading uncertainties in order to obtain a reliable design. Several categories of critical failure scenarios are considered in this study: The first category of failure scenarios is that of the relative displacement between two fracture surfaces should not exceed a critical threshold to ensure rapid healing. The second category is failure of the mini-plates which in this work is interpreted as when the yield stress within the mini-plates is reached, and the third category of failure scenarios is that of the yield stress in the mandible bone tissues should not be exceeded. Two fractured mandibles are studied under the convalescence period: Case I (a single isotropic bone tissue) and Case II (composite anisotropic bone tissues). During the fixation of the mini-plates, the drilling positions in the two cases can vary when considering a composite bone tissue mandible relative to a homogeneous bone tissue one. To show the effect of the bone anisotropy, an analytical formulation is developed as a helpful technique to analyze the effect of the mini-plate position changes. The bone properties used in the anisotropic case (Case II) are orthotropic. The results show that the reliability indices are very affected when considering the bone anisotropy.

KEYWORDS. Structural Reliability, Mandible Fractures, Mini-plate Fixation, Bone Anisotropy.
\end{abstract}

\section{Introduction}

This work is a continuation for the previous work of reliability analysis of the mini-plate fixation used in the fractured human mandibles published by Kharmanda et al. (2017a), in which the mandible is assumed to be homogenous, isotropic linear elastic bone (a single cortical tissue) and the used mini-plates are considered close to each other (Fig. 1a). However, it is well-known that the bone tissues is anisotropic as well as inhomogeneous. In this work, a more realistic model is studied and its reliability level is compared with the previous study in order to show the effect of the bone anisotropy on the reliability assessment of the mini-plate fixation system stabilization in symphysis mandibular fractures. 
The mandible fracture is a frequent bone injury and the injuries are commonly causes by vehicle accidents, falls, violence and sport. One strategy of the fractured mandible fixation is the use of mini-plates. The mini-plate fixation to the mandible is carried out by screws. Two types of plates can be found: locking and non-locking types. The locking type plates are equipped with threads such that the screws are rigidly attached to the plates. The locking plate type has the advantage of not requiring the mini-plate to be perfectly adapted to the bone while still preventing alterations in the alignment. To illustrate the mini-plate fixation strategy, a sketch of two studied mandibles shown in Figs $1 \mathrm{a}$ and $\mathrm{b}$.

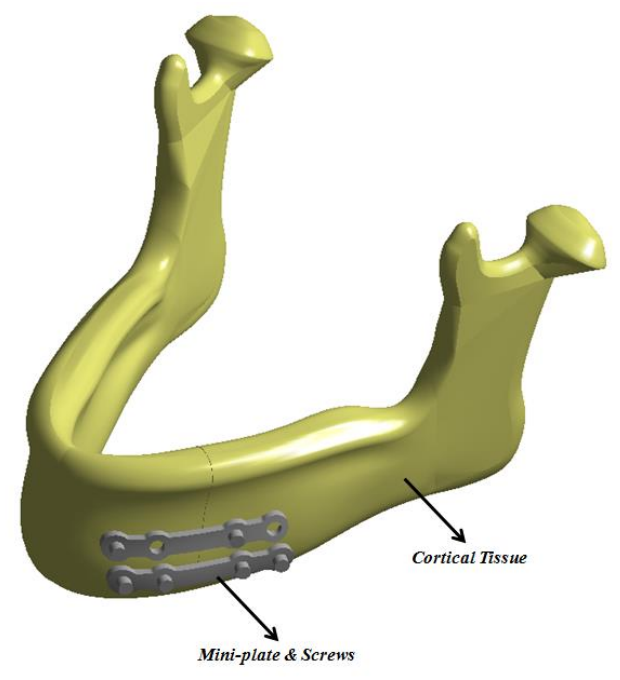

$\mathrm{a}$

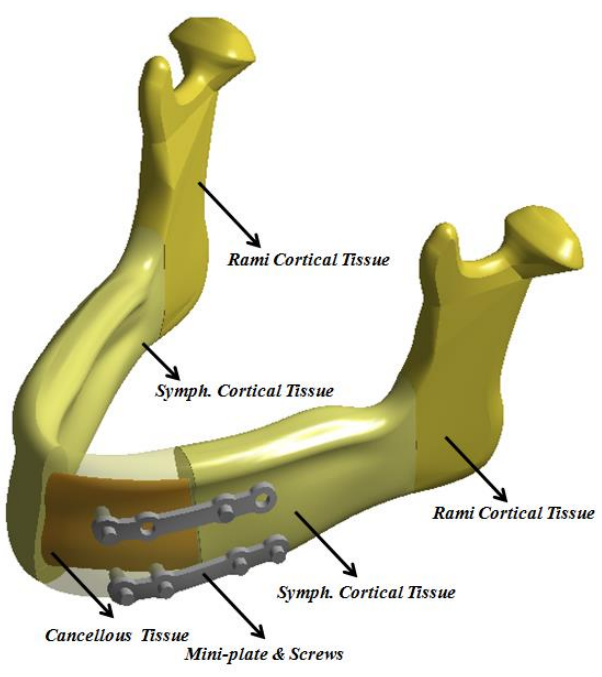

$\mathrm{b}$

Fig. 1. Two models of a mandible fracture fixation using mini-plates: a) Case I, a single isotropic bone tissue and b) Case II, composite anisotropic bone tissues

Numerical simulation is an important tool in the design process of biomechanical systems. The fixation of a mandible fracture using mini-plates was numerically investigated in Fernández et al. (2003) using finite element method. Next, a finite element investigation of mandible fracture has been carried out by Korkmaz (2007). The only load which had been considered, was the bite force, i.e. the muscle forces were ignored. Several mini-plate systems were investigated in order to provide recommendations regarding location, orientation, and type. In order to show the importance of the masseter, temporalis, lateral and medial pterygoïd forces, Mesnard et al. (2011) and Ramos et al. (2014) included them in the analysis. Kharmanda et al. (2014) showed the importance of the muscle force integration during the conceptual and detailed design stages.

In the literature, few researchers have integrated optimization and reliability into design of the systems of mini-plates used in treating fractured mandibles (Kharmanda and El-Hami 2017a; Kharmanda and El-Hami 2017c). In fact, conventional, traditional, deterministic design strategies considering uncertainties through empirical safety factors (Chakraborty and Bhattacharya 2012), which implies that the actual uncertainties in materials, geometry and loading are not truly considered. The strategy based on safety factors can result in a very conservative design as well as a design which is likely to fail. A number of uncertainties should be encountered in the design of replacement systems. These uncertainties stem from the variability of the loading and materials properties in addition to the uncertainties resulting from the geometrical modeling. The uncertainties can be classified in three main categories, namely irreducible, reducible and statistical uncertainties (Kharmanda and El-Hami 2016; Kharmanda and El-Hami 2017b). In the best-case scenario, it is difficult to completely eliminate these uncertainties, but they can be reduced or minimized. Thus, all parameters of interest in a design can be modeled as random variables (Ayyub and McCuen 2011). 
A number of uncertainties must be taken into account when designing biomechanical systems. For mandible fixation systems, geometry, bite force, muscle activity and material behavior as well as boundary conditions are in general not entirely known and simplifying assumptions are used extensively. Reliability-based methods have previously been utilized for design and assessment of biomechanical systems. Procedures based on Monte Carlo and Latin hypercube methods are common; cf. e.g. (Bah and Browne 2009). For applications where the number of random variables is large, the computational cost is high and instead FORM (First Order Reliability Methods) and SORM (Second Order Reliability Method) can be utilized (Kharmanda and El-Hami 2016).

Our previous developments can be classified according to the isotropy and anisotropy of bone tissues. Considering the bone isotropy, Kharmanda and Kharma (2016) integrated structural optimization and reliability concepts into mini-plate fixation strategy used in symphysis mandibular fractures (unilateral fracture, a clinical case of a mal patient 28 years of age) considering a single cortical tissue. The structural reliability levels were estimated for a single failure mode and multiple failure modes considering the bone tissue as an isotropic material. Next, Kharmanda et al. (2017a) performed the reliability analysis with a different failure mode considering also the bone tissue (only cortical tissue) as an isotropic material. The most important failure mode was the relative displacement between two fracture surfaces that must not exceed an allowable value. Considering that the mandible is composed of cortical and cancellous tissues, Kharmanda et al. (2017b) studied another clinical case of a fractured mandible (frontal fracture, a mal patient 35 years of age) to evaluate the reliability level in both convalescence and healing periods. The reliability evaluation leads to reasonable value in both convalescence and healing periods. However, when considering the bone anisotropy, Kharmanda et al. (2016) and Kharmanda et al. (2018) introduced the reliability analysis as a probabilistic method to predict failure in mini-plate based fixation of a mandible fracture in the early phase of the healing process following surgical operation considering both cortical and cancellous tissues. Here, an equivalent isotropic approximation is used to deal with orthotropic behaviors. This simplification may not lead to realistic evaluations of the reliability levels.

In this work, the bone anisotropy is considered because the material properties in the three directions in the mandible are different (Bonnet et al. 2009). The relative displacement between fracture surfaces and yield in plates and bone tissue are critical failure modes. Evidently, failure due to e.g. screw loosening can be included but it is out of the aim of the present study. A comparison between two cases under convalescence period is carried out in order to show the effect of the bone anisotropy on the reliability assessment. In the first case study (Case I), the bone material is assumed as cortical bone being homogenous and isotropic material, while in the second case (Case II), the bone material is composed of cortical and cancellous tissues being anisotropic. Critical failure modes are identified and the overall reliability are evaluated. It is shown that the bone anisotropy cannot be neglected in the numerical simulations and the reliability assessment.

\section{Material and methods}

\subsection{Model description}

\subsubsection{Geometry and meshing}

A numerical representation of the mandible geometry is performed using SolidWorks software and the numerical simulation and optimization procedures are performed using the ANSYS software. Due to the limited influence of the teeth on the mechanical response of the mandible, these are for simplicity ignored in the geometrical models. Fig. 1a shows the geometrical model of Case I where a single isotropic bone tissue is considered and Fig. 1b shows the geometrical model of Case II where composite anisotropic bone tissues are considered. The finite element mesh consists of 
14845 elements for Case I and 35528 elements for Case II (non-linear elements of the types: CONTACT \& SOLID). There is non-penetrating contact between the fracture surfaces. In accordance with Korkmaz (2007), two mini-plates were fixed to the bone with 6 screws as shown in Fig. 1. For simplicity two identical mini-plates were used although one 4 hole I-plate and one 2-hole I-plate could have been used. The screws were modeled as simple cylinders with a length appropriate for penetration. Standard I mini-plates were used and the dimensions of the mini-plates are shown in Fig. 2a. In accordance with the locking type mini-plate concept the interface between the screws and the plates is considered fully bonded. It is supposed that there is no contact between the mini-plate surfaces and the bone. The thickness of the mini-plates is chosen to be: $2 \mathrm{~mm}$.

\subsubsection{Material properties}

The mini-plates and the screws are assumed to be made of titanium with Young's modulus $110 \mathrm{GPa}$ and Poisson's ratio 0.34 . The yield stress of the mini-plates is assumed to be $860 \mathrm{MPa}$ (Korkmaz 2007). In Case I, the mandible is assumed to be homogenous, isotropic linear elastic with Young's modulus 14GPa and Poisson's ratio 0.3 (Korkmaz 2007). According to the relation between the yield stress and elasticity modulus of bone proposed by Kharmanda (2015), the yield stress for the cortical bone in tension is taken as $\sigma_{y}^{C o r}=80 \mathrm{MPa}$. However, in Case II, the mandible is assumed to be composed of three tissues with anisotropic behavior. All material properties are found in Table 1. According to the equivalent isotropic approximation for reliability-based design of miniplate fixation used in fractured mandibles (Kharmanda et al. 2018), the yield stress in tension for the cancellous, symphyseal cortical and ramus cortical tissues are respectively: $\sigma_{y}^{C a n}=3.3 \mathrm{MPa}$, $\sigma_{y}^{\text {Sym }}=70 \mathrm{MPa}, \sigma_{y}^{\text {Ram }}=78 \mathrm{MPa}$.

\begin{tabular}{|c|c|c|c|}
\hline \multirow{2}{*}{ Parameters } & \multirow{2}{*}{ Cancellous Tissue } & \multicolumn{2}{|c|}{ Cortical Tissues } \\
\cline { 3 - 4 } & & Sym. & Ram. \\
\hline$E_{x}(\mathrm{MPa})$ & 960 & 22900 & 17000 \\
\hline$E_{y}(\mathrm{MPa})$ & 390 & 14200 & 13800 \\
\hline$E_{z}(\mathrm{MPa})$ & 320 & 10500 & 10600 \\
\hline$v_{x y}$ & 0.3 & 0.19 & 0.38 \\
\hline$v_{y z}$ & 0.3 & 0.31 & 0.23 \\
\hline$v_{x z}$ & 0.3 & 0.29 & 0.47 \\
\hline$G_{x y}(\mathrm{MPa})$ & 170 & 6000 & 6200 \\
\hline$G_{y z}(\mathrm{MPa})$ & 130 & 3700 & 4100 \\
\hline$G_{x z}(\mathrm{MPa})$ & 90 & 4800 & 5400 \\
\hline
\end{tabular}

Table 1. Material properties used in the bone anisotropic case (Castano et al. 2002).

\subsubsection{Boundary conditions and developments}

The muscle forces being included in the model are Superficial Masseter (SM), Deep Masseter (DM), Anterior Temporalis (AT), Medial Temporalis (MT), Posterior Temporalis (PT) and Medial Pterygoid (MP) (Table 2). During the bite process, the digastric muscles are not significantly active and therefore for simplicity ignored in analysis (Ramos et al. 2014). The origin of the coordinate system is considered at the position where the bite force is applied and the y-axis is aligned with the bite force vector, cf. Fig. 2b. According to Fig. 2b, the bite force is applied in region A. The sums of 
the masseter muscle forces are $M^{\text {Right }}$ and $M^{\text {Left }}$ and applied in regions $B$ and $C$. The sums of the temporal muscle forces are $T^{\text {Right }}$ and $T^{\text {Left }}$ and applied in regions $D$ and $E$. Finally, the sums of the pterygoid muscle forces are $P^{\text {Right }}$ and $P^{\text {Left }}$ and applied in regions $F$ and $G$. The fixation of the mandible is located at in regions $H$ and $I$.

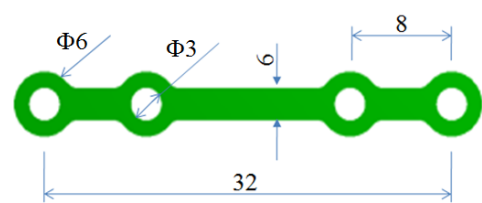

a

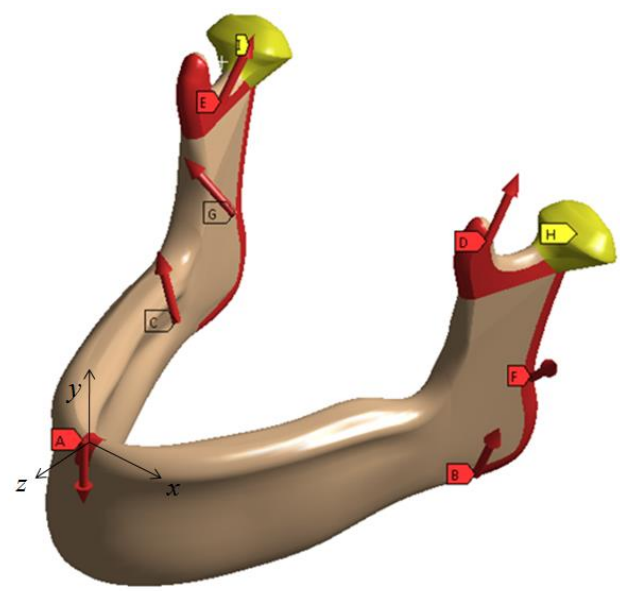

$\mathrm{b}$

Fig. 2. a) The dimensions of the used I mini-plates, and b) Illustration of the boundary conditions, different muscle forces, provided in Table 3.

After surgical operation, the patient should eat soft food that renders in low bite forces. Considering the experimental results of Kumar et al. (2013), the maximum bite force is $F_{b}=44 \mathrm{~N}$ after the surgical operation. The muscle forces presented in Table 2 are measured at its maximum capacity of an un-fractured mandible (Mesnard et al. 2011), which corresponds to a bite force of $F_{b}=208 \mathrm{~N}$. Thus, in the numerical model, the forces in Table 3 are scaled such that the bite force $F_{b}=44 \mathrm{~N}$ is obtained.

\begin{tabular}{|c|c|c|c|}
\hline Muscle Forces & $F_{x}(\mathrm{~N})$ & $F_{y}(\mathrm{~N})$ & $F_{z}(\mathrm{~N})$ \\
\hline Superficial Masseter (SM) & 18.2 & 303.3 & 12.1 \\
\hline Deep Masseter (DM) & 7.8 & 128.3 & 15.6 \\
\hline Anterior Temporalis (AT) & -18.4 & 104.8 & -43.8 \\
\hline Medial Temporalis (MT) & -6.5 & 36.3 & -53.1 \\
\hline Posterior Temporalis (PT) & -3.4 & 6.8 & -37 \\
\hline Medial Pterygoid (MP) & 187.4 & 325.1 & -76.5 \\
\hline
\end{tabular}

Table 2. Muscle forces components (Mesnard et al. 2011)

The components of the applied muscle forces in reference to the selected coordinate system are given in Table 3. This scaled state are denoted the mean muscle force state, and this corresponds to the muscle activity that would be obtained considering the bite force equal to $F_{b}=44 \mathrm{~N}$. However, in the case where a mandible fracture exists, the different muscle activities are not well known. This indicates that in order to estimate the probability of failure, the muscle activity (forces) cannot be taken as known, but must be considered as unknowns and an outcome of the analysis. Due to the existing uncertainties in the muscle forces, a reliability analysis is essential in order for a reliable and well performing fixation system to be designed. 
When fixing the mini-plates, the drilling positions in the two studied cases can be differently located because of the geometry complexity and the structural composition. In Case I, the mandible is considered as a single isotropic bone tissue, while in Case II, it is a composite bone tissue structure. This way two different parameters can affect the study: geometry and materials. To solve this confusion problem, a simple formulation is developed as a helpful technique to analyze analytically the effect of the mini-plate position changes. Then, the numerical results show the effect of the bone anisotropy.

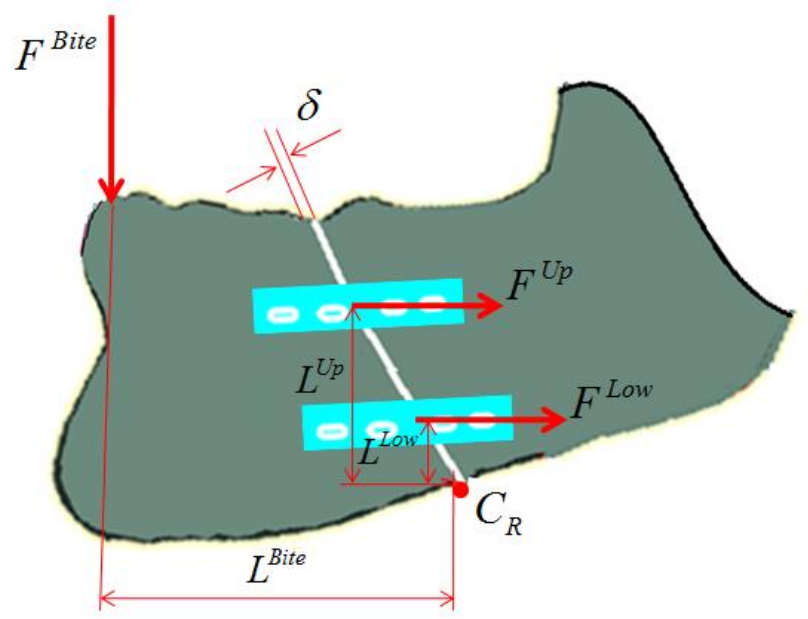

Fig. 3. 2D sketch of the different forces and related perpendicular distances between the forces and the pivot

Fig. 3 shows a 2D sketch of the different applied forces on a part of the symphyseal area. Here, the moment equilibrium equation with respect to joint $C_{R}$ (Centre of Rotation) can be written to define the relationship between the different forces. Considering the bite force $F^{\text {Bite }}$ and the reaction forces on the upper mini-plate $F^{U p}$ and on the lower mini-plate $F^{\text {Low }}$, the corresponding perpendicular distances between the forces and the joint $C_{R}$ are respectively $L^{B i t e}, L^{U p}$ and $L^{\text {Low }}$. When comparing the fixation for Case I (Fig. 1a) relative to Case II (Fig. 1b), the position of the upper mini-plate is changed. Thus, the moment equilibrium equation with respect to joint $C_{R}$ can be used to define the upper force as follows:

$$
F^{U p}=\frac{F^{\text {Bite }} \cdot L^{\text {Bite }}-F^{\text {Low }} \cdot L^{\text {Low }}}{L^{U p}}
$$

This way the effects of the geometry (drilling positions) can be analytically determined when discussing the results, while the effects of the bone anisotropy can be only numerically determined.

\subsection{Reliability analysis}

\subsubsection{Reliability concept and formulations}

In structural reliability theory, several effective techniques have been developed, namely FORM, SORM and simulation techniques (Kharmanda and El-Hami 2016). According to Hasofer and Lind (1976), the normalized space of independent Gaussian variables (Fig. 4b) is used instead of the space of physical variables (Fig. 4a). The transformation of the random variables $\mathbf{y}$ into the standard normalized space is calculated as $\mathbf{u}=T(\mathbf{y})$ considering that $T(\mathbf{y})$ is the probabilistic transformation function. 


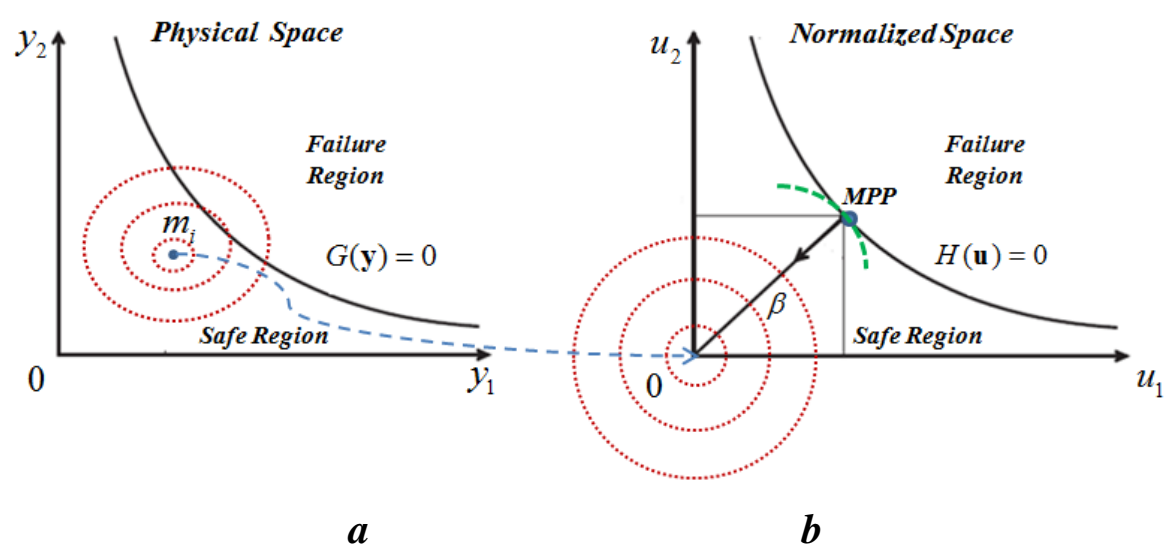

Fig. 4. a) The physical space, and b) The normalized space

The probabilistic transformation function is considered as normal distribution and it defines the normalized variable $u_{i}$ by the transformation

$$
u_{i}=\frac{y_{i}-m_{i}}{\sigma_{i}}
$$

where $y_{i}$ represents the random variable in the normalized space, with the mean values $m_{i}$ and standard-deviations $\sigma_{i}$. The standard deviations are assumed to be proportional to the mean values (Kharmanda et al. 2010). The reliability index $\beta$ is obtained from the solution to the constrained optimization problem:

$$
\beta=\min d(\mathbf{u}) \quad \text { s.t.: } \max \left(H_{i}(\mathrm{u})\right)=0 \quad i=1, . ., n
$$

where the distance is given by:

$$
d=\sqrt{\sum u_{i}^{2}}
$$

The solution to Equation (3) leads to the Most Probable failure Point (MPP) and the resulting minimum value represents the distance between the active limit state function $H(\mathbf{u})$ and the origin of the normalized space (Hasofer and Lind 1974). The probability of failure corresponding to the reliability index, is numerically computed as follows:

$$
P_{f} \approx \Phi(-\beta)
$$

where $\Phi($.$) is the standard Gaussian cumulated function expressed by:$

$$
\Phi(Z)=\frac{1}{\sqrt{2 \pi}} \int_{-\infty}^{Z} e^{-\frac{z^{2}}{2}} d z
$$

For practical structural engineering studies, Equation (5) gives a sufficiently accurate assessment of the failure probability (Kharmanda et al. 2010). In general, the failure probability $P_{f}$ in structural engineering applications should be in the range $\left[10^{-3}-10^{-5}\right]$ which corresponds to a reliability index $\beta \in[3-4.25]$ (Kharmanda and El-Hami 2016; Kharmanda and El-Hami 2017b). 
There are several scenarios that can lead to failure of the fracture healing and the first being the relative movement of the fracture surfaces in the early phase of the healing process (Perren 2002). The threshold for the relative displacement $\delta$ (Fig. 3) of the fracture surfaces is in this work set to $\delta_{t}=150 \mu \mathrm{m}$ (Søballe 1993). This threshold has previously been considered by e.g. Weinans et al. (1993) as well as by Tams et al. (2001) and Cox et al. (2003) in studies of degradable plates used to stabilize mandible fractures. Another possible source of unsuccessful fracture healing is failure of the mini-plates. Mini-plate fracture has been reported in several studies (Iizuka et al. 1991; Hammer et al. 1997; Jensen et al. 2006; Colletti et al. 2014). These kinds of failure are either due to fracture or yield of the mini-plates. Since fracture is commonly associated with prior yielding the threshold for the stresses in the mini-plates are set to the yield strength $\sigma_{y}^{\text {Met }}$ of the material. The last potential source of failure is that of the maximum allowable stress in the bone tissues being exceeded. For Case I, the threshold for the stress in the single cortical bone tissue is denoted $\sigma_{y}^{\text {cor }}$. Thus, the three failure modes can mathematically be summarized as:

$$
\begin{aligned}
& G_{1}=\delta_{\max }^{C o r}-\delta_{t} \leq 0 \\
& G_{2}=\sigma_{\max }^{M e t}-\sigma_{y}^{M e t} \leq 0 \\
& G_{3}=\sigma_{\max }^{C o r}-\sigma_{y}^{C o r} \leq 0
\end{aligned}
$$

where $\delta_{\max }^{\text {Cor }}$ is the maximum relative displacement of the cortical tissue in the fracture zone. The parameters $\sigma_{\max }^{\text {Met }}$ and $\sigma_{\max }^{\text {Cor }}$ represents the maximum value of the von-Mises effective stress in the mini-plate and cortical bone tissue, respectively. It is supposed that the failure takes place when either the constraint $G_{1} \leq 0, G_{2} \leq 0$ or $G_{3} \leq 0$ are violated. Additional failure modes can be added to Equation (7), however for the purpose considered here the failure modes in Equation (7) suffice. However, for Case II, the threshold for the stress in the cancellous, symphyseal cortical and ramus cortical bone tissues are respectively denoted $\sigma_{y}^{\text {Can }}, \sigma_{y}^{\text {Sym }}$ and $\sigma_{y}^{\text {Ram }}$. So, the six failure modes can mathematically be summarized as:

$$
\begin{aligned}
& G_{1}=\delta_{\text {max }}^{C o r}-\delta_{t} \leq 0 \\
& G_{2}=\delta_{\max }^{C a n}-\delta_{t} \leq 0 \\
& G_{3}=\sigma_{\max }^{M e t}-\sigma_{y}^{M e t} \leq 0 \\
& G_{4}=\sigma_{\max }^{C a n}-\sigma_{y}^{C a n} \leq 0 \\
& G_{5}=\sigma_{\max }^{\text {Sym }}-\sigma_{y}^{\text {Sym }} \leq 0 \\
& G_{6}=\sigma_{\max }^{\text {Ram }}-\sigma_{y}^{\text {Ram }} \leq 0
\end{aligned}
$$

where $\delta_{\max }^{C a n}$ and $\delta_{\max }^{C o r}$ are the maximum relative displacement of the cancellous and cortical tissues in the fracture zone. The parameters $\sigma_{\max }^{\text {Met }}, \sigma_{\max }^{\text {Can }}, \sigma_{\max }^{S y m}$ and $\sigma_{\max }^{\text {Ram }}$ represent the maximum value of the von-Mises effective stress in the mini-plate, cancellous, symphyseal cortical and ramus cortical bone tissues, respectively. It is supposed that the failure takes place when either the constraint $G_{1} \leq 0$, $G_{2} \leq 0, G_{3} \leq 0, G_{4} \leq 0, G_{5} \leq 0$ or $G_{6} \leq 0$ are violated. Additional failure modes can be added to Equation (8), however for the purpose considered here the failure modes in Equation (8) suffice.

On the basis of Equation (7), the reliability index problem for Case I can be expressed as follows: 


$$
\begin{aligned}
\min & : d\left(u_{i}\right)=\sqrt{\sum_{i=1}^{n} u_{i}^{2}} \\
\text { s.t. } & : H_{1}\left(u_{i}\right)=\delta_{\max }^{C o r}\left(u_{i}\right)-\delta_{t} \leq 0 \\
& : H_{2}\left(u_{i}\right)=\sigma_{\max }^{\text {Met }}\left(u_{i}\right)-\sigma_{y}^{\text {Met }} \leq 0 \\
& : H_{3}\left(u_{i}\right)=\sigma_{\max }^{\text {Cor }}\left(u_{i}\right)-\sigma_{y}^{C o r} \leq 0
\end{aligned}
$$

And on the basis of Equation (8), the reliability index problem for Case II can be expressed as follows:

$$
\begin{aligned}
\min & : d\left(u_{i}\right)=\sqrt{\sum_{i=1}^{n} u_{i}^{2}} \\
\text { s.t. } & : H_{1}\left(u_{i}\right)=\delta_{\max }^{\text {Cor }}\left(u_{i}\right)-\delta_{t} \leq 0 \\
& : H_{2}\left(u_{i}\right)=\delta_{\max }^{\text {Can }}\left(u_{i}\right)-\delta_{t} \leq 0 \\
& : H_{3}\left(u_{i}\right)=\sigma_{\max }^{\text {Met }}\left(u_{i}\right)-\sigma_{y}^{\text {Met }} \leq 0 \\
& : H_{4}\left(u_{i}\right)=\sigma_{\max }^{\text {Can }}\left(u_{i}\right)-\sigma_{y}^{\text {Can }} \leq 0 \\
& : H_{5}\left(u_{i}\right)=\sigma_{\max }^{\text {Sym }}\left(u_{i}\right)-\sigma_{y}^{\text {Sym }} \leq 0 \\
& : H_{6}\left(u_{i}\right)=\sigma_{\max }^{\text {Ram }}\left(u_{i}\right)-\sigma_{y}^{\text {Ram }} \leq 0
\end{aligned}
$$

The random variable vector $\mathbf{y}$ contains 19 components, i.e. the bite force and the 18 muscle forces are considered as random variables. Referring to (9) and (10), it can be concluded that the reliability index is related to the distance between the mean forces values and the failure load.

\section{Results}

The developed reliability strategy is utilized to evaluate the reliability level of a mini-plate fixation system considering two studied cases and the main results are reported here. In Table 3, the force components at the Mean Point (MP) are presented. The mean values and the standard deviations of the applied forces are input for this analysis. The standard deviations are assumed to be proportional to the mean point, i.e. $\sigma_{i}=k m_{i}$ where $k=0.5$. On the basis of the initial, mean, data and standard deviation the reliability algorithm is performed using the ANSYS software. 


\begin{tabular}{|c|c|c|c|c|}
\hline \multirow{2}{*}{\multicolumn{2}{|c|}{ Parameters }} & \multirow{3}{*}{$\begin{array}{l}\text { MP } \\
-44\end{array}$} & \multirow{3}{*}{$\begin{array}{c}\text { Case I } \\
\text { MPP } \\
-81.66\end{array}$} & \multirow{3}{*}{$\begin{array}{c}\text { Case II } \\
\text { MPP } \\
-53.64\end{array}$} \\
\hline & & & & \\
\hline$F^{\text {Bite }}(\mathrm{N})$ & $F_{y}^{\text {Bite }}$ & & & \\
\hline \multirow{3}{*}{$M^{R i g h t}(\mathrm{~N})$} & $M_{x}^{R i g h t}$ & 5.46 & 1.47 & 5.84 \\
\hline & $M_{y}^{R i g h t}$ & 90.64 & 57.48 & 89.81 \\
\hline & $M_{z}^{R i g h t}$ & 5.82 & 10.64 & 6.00 \\
\hline \multirow{3}{*}{$M^{\text {Left }}(\mathrm{N})$} & $M_{x}^{\text {Left }}$ & -5.46 & -5.56 & -5.90 \\
\hline & $M_{y}^{\text {Left }}$ & 90.64 & 41.50 & 73.73 \\
\hline & $M_{z}^{L e f t}$ & 5.82 & 9.51 & 5.91 \\
\hline \multirow{3}{*}{$T^{\text {Right }}(\mathrm{N})$} & $T_{x}^{R i g h t}$ & -5.94 & -6.84 & -6.13 \\
\hline & $T_{y}^{R i g h t}$ & 31.06 & 18.00 & 31.06 \\
\hline & $T_{z}^{\text {Right }}$ & -28.12 & -51.10 & -29.51 \\
\hline \multirow{3}{*}{$T^{\text {Left }}(\mathrm{N})$} & $T_{x}^{\text {Left }}$ & 5.94 & 9.45 & 5.78 \\
\hline & $T_{y}^{\text {Left }}$ & 31.06 & 46.42 & 29.62 \\
\hline & $T_{z}^{\text {Left }}$ & -28.12 & -20.57 & -26.73 \\
\hline \multirow{3}{*}{$P^{R i g h t}(\mathrm{~N})$} & $P_{x}^{\text {Right }}$ & 39.35 & 45.44 & 59.03 \\
\hline & $P_{y}^{\text {Right }}$ & 68.27 & 75.48 & 65.95 \\
\hline & $P_{z}^{R i g h t}$ & -16.07 & -15.74 & -15.07 \\
\hline \multirow{3}{*}{$P^{\text {Left }}(\mathrm{N})$} & $P_{x}^{\text {Left }}$ & -39.35 & -42.66 & -54.69 \\
\hline & $P_{y}^{\text {Left }}$ & 68.27 & 56.88 & 66.84 \\
\hline & $P_{z}^{L e f t}$ & -16.07 & -19.13 & -16.33 \\
\hline
\end{tabular}

Table 3. The forces values for the MP and the MPP.

For Case I, a first observation that can be made is that at the MPP state, the symmetry with respect to the $x=0$ plane is broken, as an example $M_{y}^{\text {Left }}$ are significantly lower than $M_{y}^{\text {Right }}$ at the MPP. It can also be concluded that the bite force is almost double compared to the mean force level but still approximately a factor of three less than the bite force at the fully operating state. The resulting maximum von-Mises stresses at the MP and at the MPP in the different parts are presented in Table 4. The simulations using the mean forces results in the maximum von-Mises stress being $52 \mathrm{MPa}$ and it is located in the lower mini-plate, cf. Fig. 5. At the MPP state the maximum vonMises stress is $65 \mathrm{MPa}$ which clearly indicates that there is a large safety marginal to yield in the mini-plates. The maximum von-Mises stress for the mean forces in the bone is $19 \mathrm{MPa}$. When the MPP is found the maximum von-Mises stress in the bone is $29 \mathrm{MPa}$, also indicating that there is a marginal to the stress level in the bone for the chosen conditions. From the analysis using the mean force level, it can be concluded that the relative displacement at the fracture surfaces is $\delta_{\max }^{C o r}=131 \mu \mathrm{m}$. For the MPP state, it can be concluded that $\delta_{\max }^{\text {Cor }}-\delta_{t} \approx 0$ is fulfilled and as a result the relative motion between the fracture surfaces constrain the design. The computed reliability index 
for the considered force variability equals $\beta=4.17$ which corresponds to failure probability $P_{f} \approx 1.6 \times 10^{-5}$, cf. Equation (5).

\begin{tabular}{|cc|c|c|c|c|}
\hline \multirow{2}{*}{ Layers } & Parameters & \multicolumn{2}{|c}{ Case I } & \multicolumn{2}{c|}{ Case II } \\
\cline { 3 - 6 } Cortical & $\delta_{\max }^{\text {Cor }}(\mu \mathrm{m})$ & 131 & 149.1 & 132 & 148.4 \\
\hline Cancellous & $\delta_{\max }^{\text {Can }}(\mu \mathrm{m})$ & --- & --- & 116 & 124.5 \\
\cline { 2 - 6 } Metal & $\sigma_{\max }^{\text {Up }}(\mathrm{MPa})$ & $\mathbf{3 3 . 5 1}$ & $\mathbf{4 8 . 8 3}$ & $\mathbf{3 5 . 9 4}$ & $\mathbf{5 0 . 9 0}$ \\
\cline { 2 - 6 } & $\sigma_{\max }^{\text {Low }}(\mathrm{MPa})$ & $\mathbf{5 1 . 8 4}$ & $\mathbf{6 5 . 0 4}$ & $\mathbf{6 4 . 4 5}$ & $\mathbf{9 2 . 7 5}$ \\
\hline \multirow{2}{*}{$\begin{array}{c}\text { Sym. } \\
\text { Cortical }\end{array}$} & $\sigma_{\max }^{\text {Right }}(\mathrm{MPa})$ & $\mathbf{1 7 . 7 8}$ & $\mathbf{2 9 . 3 4}$ & 6.28 & 11.75 \\
\hline \multirow{2}{*}{ Ram. } & $\sigma_{\max }^{\text {Left }}(\mathrm{MPa})$ & $\mathbf{1 9 . 0 7}$ & $\mathbf{2 6 . 0 7}$ & 12.63 & 16.69 \\
Cortical & $\sigma_{\max }^{\text {Right }}(\mathrm{MPa})$ & --- & --- & $\mathbf{1 8 . 3 6}$ & $\mathbf{2 9 . 7 2}$ \\
\hline \multirow{2}{*}{ Cancellous } & $\sigma_{\max }^{\text {Left }}(\mathrm{MPa})$ & --- & --- & $\mathbf{2 2 . 6 2}$ & $\mathbf{2 2 . 5 2}$ \\
\cline { 2 - 6 } & $\sigma_{\max }^{\text {Right }}(\mathrm{MPa})$ & --- & --- & 0.24 & 0.38 \\
\hline & $\sigma_{\max }^{\text {Left }}(\mathrm{MPa})$ & --- & --- & 0.16 & 0.25 \\
\hline & $\beta$ & --- & 4.17 & --- & 1.5 \\
\hline & $P_{f}$ & --- & $1.6 \times 10^{-5}$ & --- & $6.7 \times 10^{-2}$ \\
\hline
\end{tabular}

Table 4. The von-Mises stress and relative displacement values at the MP and the MPP.

For Case II, a first observation that can be made is that at the MPP state, the symmetry with respect to the $x=0$ plane is not broken similarly to Case I. Only $M_{y}^{\text {Left }}$ are lower than $M_{y}^{\text {Right }}$ at the MPP. The bite force is bigger than the mean force level almost by $20 \%$. The resulting maximum von-Mises stresses at the MP and at the MPP in the different parts are presented in Table 4. The simulations using the mean forces results in the maximum von-Mises stress being $65 \mathrm{MPa}$ and it is located in the lower mini-plate. At the MPP state the maximum von-Mises stress is $93 \mathrm{MPa}$ which clearly indicates that there is a large safety marginal to yield in the mini-plates. The maximum vonMises stress for the mean forces in the ramus cortical bone is $23 \mathrm{MPa}$. When the MPP is found the maximum von-Mises stress in the ramus cortical bone is $30 \mathrm{MPa}$, also indicating that there is a marginal to the stress level in the bone for the chosen conditions. From the analysis using the mean force level, it can be concluded that the relative displacement at the fracture surfaces is $\delta_{\max }^{C o r}=132 \mu m$. For the MPP state, it can be concluded that $\delta_{\max }^{C o r}-\delta_{t} \approx 0$ is fulfilled and as a result the relative motion between the fracture surfaces constrain the design. The computed reliability index for the considered force variability equals $\beta=1.50$ which corresponds to failure probability $P_{f} \approx 6.7 \times 10^{-52}$, cf. Equation (5). Fig. 5 shows a comparison between the maximum vonMises stresses when considering the bone isotropy and anisotropy in the mean point and the MPP. 


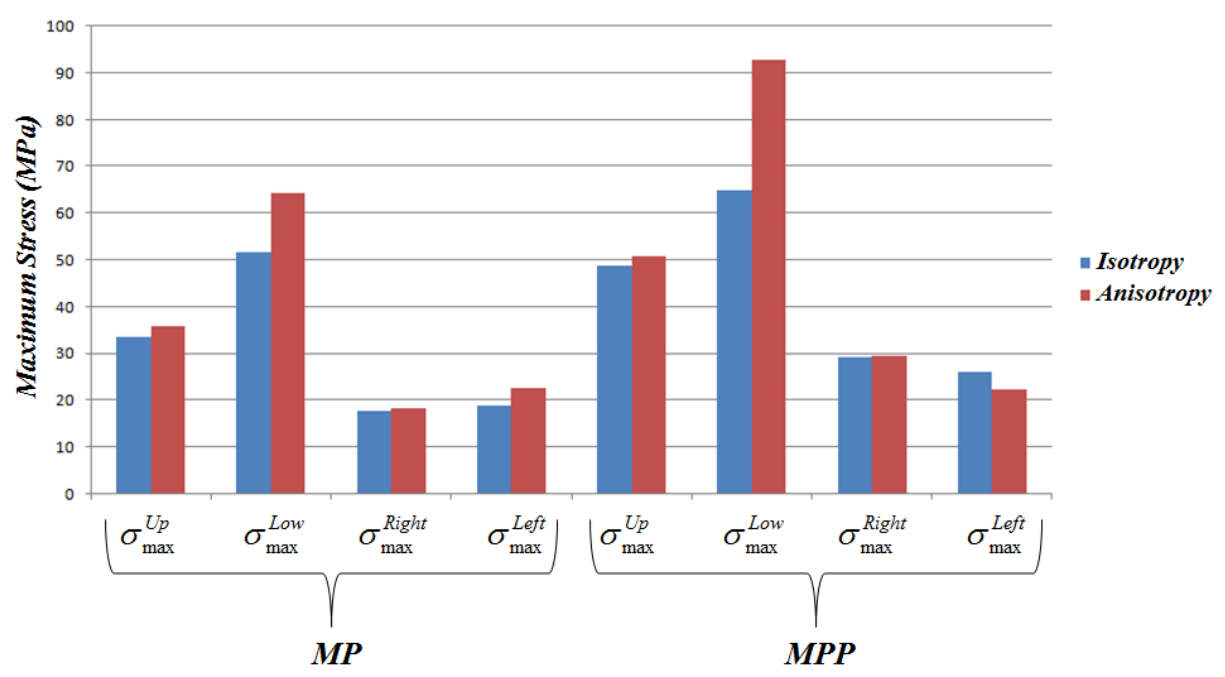

Fig. 5. Comparison between the maximum von-Mises stresses when considering the bone isotropy and anisotropy.

\section{Discussion and conclusion}

During surgical operation, some muscles can be cut or harmed which leads to that it cannot be expected that all muscles are fully functional. In addition to the uncertainties associated with the state of the muscles, the geometry and material properties of the mandible generally varies with e.g. gender and age. As a consequence of the multiple causes of uncertainties, it can be concluded that purely deterministic analysis methods are not suitable for design of fracture management systems.

Several sources of failure of the fracture healing process exist. It is for instance required that the maximum stress in both bone and metallic components must not exceed prescribed limits since it may introduce plasticity causing low cycle fatigue or fracture. Furthermore, to facilitate restoration of the bone at the fractured interface, the relative displacement between the fracture surfaces must not exceed an allowable value, in the present work this threshold is chosen to be $\delta_{t}=150 \mu \mathrm{m}$. It can be concluded that for the mean forces, no failure mode is active (Case I and Case II).

The reliability formulations (9 and 10) presented herein are general and they can easily be extended to take other design constraints into account. One such additional failure mechanism is for instance screw loosening which might lead to failure of the healing process. It has been demonstrated that the developed reliability algorithm is capable of identifying the critical failure mode from the set of potential failure modes. This information is valuable and useful since it can be used to set the direction of further development of the fixation system. For the specific mini-plate system considered herein, it can be concluded that failure most likely occurs due to violation of the maximum relative displacement constraint. Moreover, for the specific situation it can also be concluded that the stress level in the mini-plates are not limiting the design. As a consequence, a plate material with lower yield stress but higher Young's modulus is favorable since the overall reliability is increased for such alternative material.

Another interesting observation from the reliability analysis is that the MPP is found for a state where the muscle forces are non-symmetric, especially for Case I. Such non-symmetric states can be triggered by non-symmetric loads or non-symmetric muscle activities which are very likely to occur as results of the surgery. In a conventional, deterministic, analysis such effects are difficult to include in a systematic manner.

The strategy outlined in Case I (Kharmanda et al. 2017a) is general but it includes several simplifications. However, Case II presented in this work includes more realistic conditions such as 
bone anisotropy. The numerical model used in Kharmanda et al. (2017a) serves the purposes of illustrating the integration of the reliability concept. The numerical simulations of the mean points show that the anisotropic behavior of the mandibular tissues cannot be neglected in the numerical simulations. A similar conclusion can be found in the works of Bonnet et al. (2009) and Bonnet et al. (2013) where a three-dimensional finite element model of a mandible with a prosthesis supported by four implants has been developed. They concluded that to neglect the bone anisotropy influence can affect the loading transfer. This result is highly demonstrated in this paper where the probability of failure highly increased. To show the effect of the bone anisotropy, the analytical formulation (1) shows that when increasing the distance $L^{U p}$ for Case II as shown in Fig. 1b relative to Case I (Fig. 1a), the corresponding force $F^{U p}$ must be decreased and the stress value in the upper mini-plate must also be decreased. However, Table 4 shows that the maximum stresses in the upper and lower mini-plates increase for the MP which is considered a direct simulation for both cases (Case I and Case II). This means that the bone anisotropy affect even the direct simulation of the MP (given point). This influence becomes much more significant when reaching the MPP (see Fig. 5).

There are several limitations of the present study. One important limitation is the material law for the bone, which in the present work is assumed to be time independent. Moreover, the bite force, muscle forces and boundary conditions are evaluations which are based on scaling of measurements on muscles performed at full operation. Furthermore, the variations of the forces are considered to be normally distributed and to improve the accuracy, the quality of the statistical data should be improved. It is, however, interesting that although the analysis suffers from incomplete input data, it provides an indication of which failure mode that is the most critical. In the future work, a six sigma methodology can be integrated in order to determine the extent to which uncertainties in the model affect the results.

\section{References}

Ayyub, B.M.; McCuen, R.H. (2011): Probability, Statistics and Reliability for Engineers and Scientists, CRC Press, Taylor \& Francis Group, 2011.

Bah, M.T.; Browne, M. (2009): Effect of geometrical uncertainty on cemented hip implant structural integrity. Journal of Biomechanical Engineering, 131(5), 054501.

Bonnet, A.S.; Postaire, M.; Lipinski, P. (2009): Biomechanical study of mandible bone supporting a four-implant retained bridge: Finite element analysis of the influence of bone anisotropy and foodstuff position, Medical Engineering \& Physics 31 (2009) 806-815.

Bonnet, A.S.; Lipinski, P.; Creuillot, V. (2013): Simulations par éléments finis de solutions prothétiques mandibulaires sur implants Considérations biomécaniques et stratégies de modélisation, Réalités cliniques: revue européenne d'odontologie 24(1):67-74, January 2013.

Castano, M.C.; Zapata, U.; Pedroza, A.; Jaramillo, J.D.; Roldan, S. (2002): Creation of a three dimensional model of the mandible and the TMJ in vivo by means of the finite element method. Int J Comput Dent 2002;5:87-99.

Chakraborty, S.; Bhattacharya, G. (2012): Proceedings of the International Symposium on Engineering under Uncertainty: Safety Assessment and Management (ISEUSAM - 2012), Springer Science \& Business Media.

Colletti, G.; Battista, V.M.A.; Allevi, F.; Giovanditto, F.; Rabbiosi, D.; Biglioli, F. (2014): Extraoral approach to mandibular condylar fractures: our experience with 100 cases. Journal of Cranio-Maxillofacial Surgery, 42(5), e186-e194.

Cox, T.; Kohn, M.W.; Impelluso, T. (2003): Computerized analysis of resorbable polymer plates and screws for the rigid fixation of mandibular angle fractures. Journal of Oral and Maxillofacial Surgery, 61,481-487.

Fernández, J.R.; Gallas, M.; Burguera, M.; Viano, J.M. (2003): A three-dimensional numerical simulation of mandible fracture reduction with screwed miniplates. Journal of Biomechanics, 36(3), 329-337.

Hammer, B.; Schier, P.; Prein, J. (1997): Osteosynthesis of condylar neck fractures: a review of 30 patients. British Journal of Oral and Maxillofacial Surgery, 35(4), 288-291. 
Hasofer, A.M.; Lind, N.C. (1974): An exact and invariant first order reliability format. Journal of Engineering Mechanics (ASCE), 100, 111-121.

Iizuka, T.; Lindqvist, C.; Hallikainen, D.; Mikkonen, P.; Paukku, P. (1991): Severe bone resorption and osteoarthrosis after minplate fixation of high condylar fracture. Oral Surgery, Oral Medicine, Oral Pathology and Oral Radiology. $72,400-407$.

Jensen, T.; Jensen, J.; Nørholt, S.E.; Dahl, M.; Lenk-Hansen, L.; Svensson, P. (2006): Open Reduction and Rigid Internal Fixation of Mandibular Condylar Fractures by an Intraoral Approach: A Long-Term Follow-Up Study of 15 Patients, Journal of Oral and Maxillofacial Surgery 64,1771-1779.

Kharmanda, G.; El-Hami, A.; Souza de Cursi, E. (2010): Reliability-Based Design Optimization, In: Breitkopt, P., Coelho R.F., Multidisciplinary Design Optimization in Computational Mechanics, Chapter 11, Wiley \& Sons.

Kharmanda, G.; Kharma, M.Y.; Ristinmaa, M.; Wallin, M. (2014): Structural optimization of mini-plates in fixation of human mandible fractures, 27th Nordic Seminar on Computational Mechanics - NSCM-27, (Eds. A. Eriksson, A. Kulachenko, M. Mihaescu and G. Tibert (Eds.) KTH, Stockholm, Sweden; October 22-24, 2014.

Kharmanda, G. (2015): Reliability analysis for cementless hip prosthesis using a new optimized formulation of yield stress against elasticity modulus relationship, Materials and Design 65, 496-504.

Kharmanda, G.; Ibrahim, M.H.; El-Hami A. (2016): Reliability analysis of mini-plate stabilization used in human fractured mandibles, 22d ESB conference, July 10, 2016, Lyon, France.

Kharmanda, G.; Kharma, M.Y. (2016): Evaluating the Effect of Minimizing Screws on Stabilization of Symphysis Mandibular Fracture by 3D Finite Element Analysis, Journal of Maxillofacial and Oral Surgery, 16(2), pp 205-211. DOI: 10.1007/s12663-016-0903-9.

Kharmanda, G.; El-Hami, A. (2016): Reliability in Biomechanics, ISTE \& Wiley, ISBN: 9781786300249, pp 266, November 2016.

Kharmanda, G.; El-Hami, A. (2017a): Biomechanics: Optimization, Uncertainties and Reliability, ISTE \& Wiley, ISBN: 9781786300256, pp 254, January 2017.

Kharmanda, G.; Kharma, M.Y.; El-Hami A. (2017a): Integration of reliability concept into orthodontic prosthesis design: Application on mini-plate fixation systems used in fractured mandibles, Journal of Uncertainties and Reliability of Multiphysical Systems, 17-1, January 2017.

Kharmanda, G.; El-Hami, A. (2017b): Fiabilité en biomécanique : Principes \& Analyses, ISTE publication, London, ISBN : 978-1-78405-274-4, pp 252, May 2017.

Kharmanda, G.; El-Hami, A. (2017c): Fiabilité et optimisation structurale en biomécanique : Méthodologies \& Applications, ISTE publication, ISBN : 978-1-78405-250-8, May 2017.

Kharmanda, G.; Shokry, A.; Kharma, M.Y. (2017b): Integration of reliability analysis into mini-plate fixation strategy used in human mandible fractures: Convalescence and healing periods, In : Acta of Bioengineering and Biomechanics. 19, 4, pp 13-23.

Kharmanda, G.; Kharma, M.Y.; El-Hami A. (2018): An equivalent isotropic approximation for reliability-based design of mini-plate fixation used in fractured mandibles, Journal of Uncertainties and Reliability of Multiphysical Systems, 18-2 (1), May 2018.

Korkmaz, H.H. (2007): Evaluation of different mini-plates in fixation of fractured human mandible with the finite element method, Oral Surgery, Oral Medicine, Oral Pathology and Oral Radiology, 103,e1-e13.

Kumar, S.T.; Saraf, S.; Devi, S.P. (2013): Evaluation of Bite Force After Open Reduction and Internal Fixation Using Microplates, Journal of Dentistry, Tehran University of Medical Sciences, 10 (5), 466-477.

Mesnard, M.; Ramos, A.; Ballu, A.; Morlier, J.; Cid, M.; Simoes, J.A. (2011): Biomechanical analysis comparing natural and alloplastic temporomandibular joint replacement using a finite element model, Journal of Oral and Maxillofacial Surgery, 69(4), 1008-1017.

Perren, S.M. (2002): Evolution of the internal fixation of long bone fractures. The scientific basis of biological internal fixation: Choosing a new balance between stability and biology. The Journal of Bone Joint Surgery, 84- B, 10931110.

Ramos, A.; Marques, H.; Mesnard, M. (2014): The effect of mechanical properties of bone in the mandible, a numerical case study, Advances in Biomechanics and Applications, 1(1), 067-076. 
Søballe, K. (1993): Hydroxyapatite ceramic coating for bone implant fixation. Acta Ortopaedica. 64:1, 1-58.

Tams, J.; van Loon, J.-P.; Otten, B.; Bos, R.; (2001): A computer study of Biodegradable plates for internal fixation of mandible fractues. Journal of Oral and Maxillofacial Surgery, 59. 404-407.

Weinans, H.; Huiskes, R.; Grootenboer, H. J. (1993): Quantitative analysis of bone reactions to relative motions at implant-bone interfaces. Journal of Biomechanics, 26(11), 1271-1281. 